\title{
Multi-Feature Ensemble with Support Vector Machine Classification for CBIR Models
}

\author{
Paru Sharma1, Dr.Raman Chadha2 \\ ${ }^{I}$ Research Scholar, M. Tech (CSE), \\ ${ }^{2}$ Professor, Head (CSE), \\ ${ }^{1,2}$ CGC Technical Campus, Jhanjeri, Mohali,
}

\begin{abstract}
The complex image matching procedure on the large image data volume require a lot of processing power and storage space, which makes the cloud platforms very much adaptable for such CBIR systems. The cloud computing enables the capability of the CBIRs to expand the computing and storage resources as the requirement rises above the registered scale. The greatest challenge rises in the CBIRs when it comes to search the large volumes of encrypted image data. The image data is stored in the encrypted to protect the privacy of the data and to protect it from various information stealing attacks on the web storage resources. The proposed model has been designed in the layered approach, where the image features are matched one after one to prepare the final image ranking. The semantic memory has been also utilized in the form of semantic memory with fuzzy sets and user feedback. The semantic feature has been added to provide the capability to permanently filter the images marked by the users for the specific queries. The proposed model performance has been evaluated in the form of various performance parameters of accuracy, ranking/index building time, encryption/decryption times, semantic library lookup time, precision, recall, etc. The experimental results have proved the efficiency and robustness of the proposed model and proved it as the effective CBIR system.
\end{abstract}

Keywords: SIFT, HOG, TAMURA, SVM, CBIR

\section{Introduction}

Content based image retrieval is also called query by image content is the model for searching digital images. It is the process of retrieving desired images from the large databases. The need for the CBIR is growing rapidly because of the digital image database is increasing in size, professional needs and difficulty in locating images across the web. The meaning of content-based is searching the actual contents of the image. The image can be searched on the basis of color, Local shape and texture. The similarity among the color can be achieved by computing color histograms. Shapes are determined by the process of segmentation or edge detection. Texture can be judged by Tamura and SIFT feature descriptors. Presently this technique is used by Google. The components of CBIR model are-query image, image database, feature extraction, image matching and resultant retrieved images. There are two types of CBIR models namely general and application specific.

\section{Related Work}

Gudivada et al.[4] proposed a strong algorithm as it can manage interpretation, scale, and rotation fluctuations in images. The calculations obtained have quadratic time unpredictability regarding the aggregate number of articles in both the database and inquiry pictures.

Smeulders et al.[9] Presents a survey of 200 references in substance based picture recovery. The paper begins with examining the working states of substance based recovery: examples of utilization, sorts of pictures, the part of semantics, and the tangible crevice.

Lu et al. [7] suggested that for image recovery, relevance feedback is an intense method and has been a dynamic exploration for couple of years. Different parameter estimation procedures have been proposed for important input. Furthermore, it has figured various strategies for performing the improvement on multilevel picture substance model.

Xin et al. [10] made use of Gaussian mixture model for the representation of user's distribution of target which has narrowed down the gap between high level and low level features. A system is proposed to determine client's contention input, since current image recovery frameworks are unequipped for catching client's conflicting aims.

Krishnapuram et al. [5] have proposed FIRST i.e. Fuzzy image retrieval system which uses the fuzzy set theory to represent an image, similarity measure and relevance feedback. FIRST incorporates these ideas. FIRST make use of attributes, spatial relations and linguistic queries to handle the exemplary based graphical sketches. Fuzzy attributes relational graphs are used to represent the images.

Liu et al.[6] keeping in mind the end goal to enhance the recovery exactness of substance based picture recovery frameworks, examination center has been moved from planning modern low-level highlight extraction 
calculations to diminishing the 'semantic hole' between the visual highlights and the abundance of human semantics. This paper endeavors to give a thorough overview of the late specialized accomplishments in abnormal state semantic-based picture recovery.

Yang et al. [11] NIR is an open source distributed computing empowered substance based picture recovery framework. With the improvement and promotion of distributed computing, more specialists from diverse exploration ranges do research with the assistance of distributed computing. This paper exhibits our thoughts, discoveries, outline and the framework from our work of NIR.

Chang et al. [2] analyzed the contents of image and suggested that retrieval of semantics is important during semantic based image retrieval. PCA (principal component analysis) is applied to extract the image features and then concatenate them with Fuzzy-ARTNN.

Dillon et al. [3] discussed that Cloud will reshape the entire industry as a revolution. In this paper, aim is to discuss the challenges and issues of Cloud computing. First two related computing paradigms - ServiceOriented Computing and Grid computing are discussed and also their relationships with Cloud computing.

Cao et al [1] suggested that for the purpose of protecting data privacy, data has to be in the encrypted form before outsourcing it to the cloud. It replaces the traditional technique of data utilization based on plaintext keyword search. Work done in this paper focuses on the multi- keyword search over encrypted cloud data. . For the future work authors have suggested schemes to reduce overhead over computation and communication.

\section{Experimental Design}

The content based image retrieval (CBIR) models are utilized for the discovery of the similar images against the input query image by analyzing the image features in the form of texture, color, pattern etc. Most of the CBIR models utilize the color or texture based models in order to realize the image retrieval systems. Each of the feature descriptor, either based upon color or texture etc, carries some problems with the different types of the images. The idea behind the proposed model relies upon the multi- feature ensemble to increase the overall accuracy in order to improve the overall retrieval rate. Various ensemble based proposed models have proved to be efficient in the different data mining models, which stays as the primary reason behind the multi-feature ensemble based CBIR model. In this paper, we have proposed the model using the robust texture feature descriptors of Tamura and Scale Invariant Feature Transform (SIFT) along with the color based histogram of oriented gradients $(\mathrm{HoG})$ along with the SVM classification. The working of the feature descriptors have been elaborated in the following algorithms:
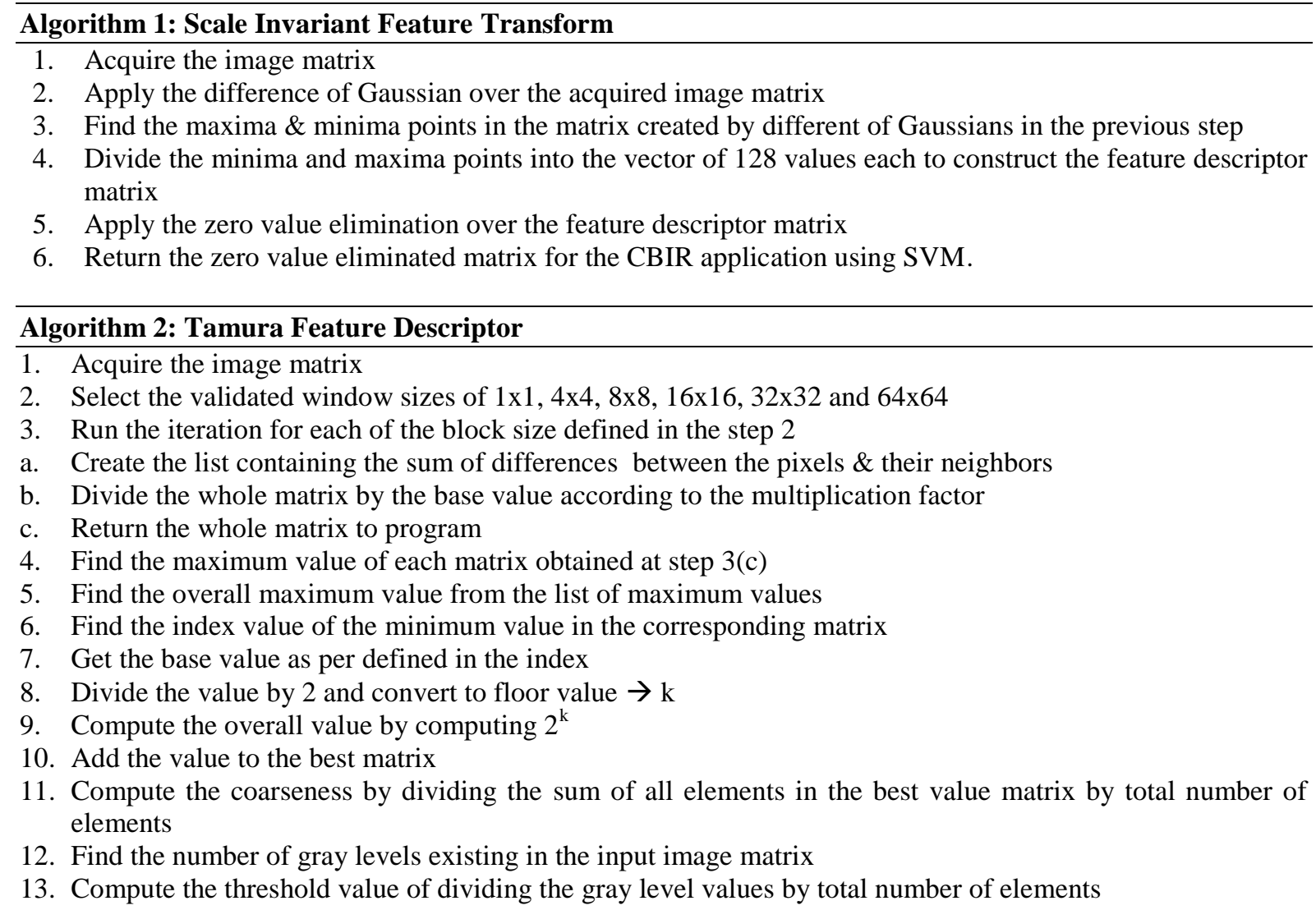
14. Compute the sum of matrix after multiplying the gray levels matrix with the threshold value matrix computed on step 13

15. Compute the overall sum of average value matrix computed on step 14 and gray levels

16. Compute the standard deviation of the matrix returns from step 15

17. Compute the value of alpha by dividing the value from step 14 by square of standard deviation

18. Compute the value of contrast by dividing the square root value of standard deviation (Step 16) by quadruple exponential of alpha (step 17)

19. Initialize the horizontal and vertical initial matrices

20. Compute the horizontal variance

21. Compute the vertical variance

22. Fit the equation by diving the sum of absolute values of horizontal and vertical variance by 2

23. Compute theta with arc tangent function over the vertical and horizontal coefficients

24. Initialize the threshold constants of Phi and Elimination Threshold

25. Compute the directionality

26. Return the value of coarseness, contrast and directionality

Algorithm 3: Histogram of Oriented Gradients (HoG)

1. Acquire the image matrix

2. Initialize the gray level vector ranging from values between 0 and 255

3. Iterate from 0 to 255

a. Find the count of current value

b. Update the count vector on the corresponding position

4. Compile the output matrix by joining the gray level vector with counts vector

5. Return the histogram of oriented gradient matrix

\section{Algorithm 4: Main Algorithm}

1. Read the query image and load in the memory as the 3-D image matrix and convert it to the grayscale to produce the 2-D image matrix.

2. The pre-processing methods are applied to remove/reduce the noise in the image

3. Perform the feature extraction over the query image to fetch the known features form the image matrix

4. Reshape the feature descriptor data for the validation of the training set containing the feature descriptors.

5. Create the group matrix with the appropriate group ids denoted to the training samples.

6. Run the SVM over the training dataset to match the testing set to obtain the training matrix in the form of similarity and bias.

7. Then the SVM classifier is applied over the SVM training data to classify the given sample.

8. Return the matching sample one the basis of SVM classification information in the classification matrix.

9. Return the decision logic.

\section{Result Analysis}

Assuming the positive evaluation set is a trustworthy representation of real world images in the content based image retrieval dataset and arranged in the form of different sized images which includes the grayscale, colored or binary images. The image retrieval dataset has been obtained from Professor Wang's official blog and contains multiple category and multi-color scale images classified in the different categories of beach, monuments, urban transport, digital and tribal images, whereas different numbers of images have been kept under the image different categories to form the final training image set. The proposed multi-stage hierarchical CBIR image dataset has been defined with the sensitive image data to test the real-time accuracy of the CBIR system. The proposed model has been found accurate with the accuracy level of more than $85 \%$ in comparison with the existing models using the different feature descriptors and classifiers.

Table 1: The table of properties calculated from the hypothesis of statistical type-1 and type-2 errors on the Category-A testing dataset

\begin{tabular}{|l|l|}
\hline PROPERTY NAME & Proposed Model \\
\hline Positive & 113 (TP 106, FP 7) \\
\hline Negative & 18 (TN 5, FN 13) \\
\hline Sensitivity & $89.08 \%$ \\
\hline Specificity & $41.67 \%$ \\
\hline Positive Likelihood Ratio & 1.53 \\
\hline Negative Likelihood Ratio & 0.26 \\
\hline Prevalence & $90.84 \%$ \\
\hline Positive Predictive Value & $93.81 \%$ \\
\hline Negative Predictive Value & $27.78 \%$ \\
\hline
\end{tabular}


131 images from various categories among the test set has been obtained for the testing of the CBIR system from the given training dataset of almost 1000 images of different categories. The training data has been used for testing the fully automated proposed image recognition and retrieval system. Some of the image taken under the consideration are the clean and noise free images, whereas some of the images with the different noise levels has been shortlisted for the testing of the proposed CBIR system

\section{Conclusion}

The trustworthiness of the proposed content based image retrieval system has been evaluated in the form of various statistical performance measure performance parameters calculated from the statistical type I and type II errors. The various types of images have been evaluated in the Professor Wang's database, which has been used for the purpose of performance evaluation of the proposed CBIR system. The database has been divided into the various categories such as tribal, beach, historical monument, urban transport and digital images of dainasaurus. The 2-stage proposed content based image retrieval system has been designed to offer the maximum accuracy in comparison with the evaluated existing techniques. The 131 images has been classified for the testing purposes and tested over the training set of 1000 images. The proposed model is the background aura based scheme, which has outperformed the existing model on the basis of various performance parameters. The proposed model have marked the Sensitivity of the almost $89.08 \%$ against the $82.45 \%$ by Early Pruning Model, $81.22 \%$ by SIFT with SVM and $73.78 \%$ against the SASI with SVM based CBIRs. The specificity of the proposed model has been recorded at nearly $41 \%$ in comparison with the $35 \%, 40 \%, 40.73 \%$ offered by SIFT, SASI and Early pruning model.

\section{References}

[1]. Cao, N., Wang, C., Li, M., Ren, K., \& Lou, W. (2014). Privacy-preserving multi-keyword ranked search over encrypted cloud data. Parallel and Distributed Systems, IEEE Transactions on, 25(1), 222-233.

[2]. Chang, C. Y., Wang, H. J., \& Jian, R. H. (2010, July). Semantic image retrieval with Fuzzy-ART. In System Science and Engineering (ICSSE), 2010 International Conference on (pp. 69-74). IEEE.

[3]. Dillon, T., Wu, C., \& Chang, E. (2010, April). Cloud computing: issues and challenges. In Advanced Information Networking and Applications (AINA), 2010 24th IEEE International Conference on (pp. 27-33). Ieee.

[4]. Gudivada, V. N., \& Raghavan, V. V. (1995). Design and evaluation of algorithms for image retrieval by spatial similarity. ACM Transactions on Information Systems (TOIS), 13(2), 115-144.

[5]. Krishnapuram, R., Medasani, S., Jung, S. H., Choi, Y. S., \& Balasubramaniam, R. (2004). Content-based image retrieval based on a fuzzy approach. Knowledge and Data Engineering, IEEE Transactions on, 16(10), 1185-1199.

[6]. Liu, Y., Zhang, D., Lu, G., \& Ma, W. Y. (2007). A survey of content-based image retrieval with high-level semantics. Pattern Recognition, 40(1), 262-282.

[7]. Lu, Y., Zhang, H., Wenyin, L., \& Hu, C. (2003). Joint semantics and feature based image retrieval using relevance feedback. Multimedia, IEEE Transactions on, 5(3), 339-347.

[8]. MOHANA, R. M., \& REDDY, A. R. M. CCBIR: A Cloud Based Implementation of Content Based Image Retrieval.

[9]. Smeulders, A. W., Worring, M., Santini, S., Gupta, A., \& Jain, R. (2000). Content-based image retrieval at the end of the early years. Pattern Analysis and Machine Intelligence, IEEE Transactions on, 22(12), 1349-1380.

[10]. Xin, J., \& Jin, J. S. (2003, December). Learning from user feedback for image retrieval. In Information, Communications and Signal Processing, 2003 and Fourth Pacific Rim Conference on Multimedia. Proceedings of the 2003 Joint Conference of the Fourth International Conference on (Vol. 3, pp. 1792-1795). IEEE.

[11]. Yang, Z., Kamata, S. I., \& Ahrary, A. (2009, November). NIR: Content based image retrieval on cloud computing. In Intelligent Computing and Intelligent Systems, 2009. ICIS 2009. IEEE International Conference on (Vol. 3, pp. 556-559). IEEE. 